BULL. AUSTRAL. MATH. SOC.

VOL. $21(1980), 175-188$.

\title{
COMPARISON THEOREMS OF HILLE-WINTNER TYPE FOR THIRD ORDER LINEAR DIFFERENTIAL EQUATIONS
}

\section{ERBE}

Integral comparison theorems of Hille-Wintner type of second order linear equations are shown to be valid for the third order linear equation $y^{\prime \prime \prime}+q(t) y=0$.

\section{Introduction}

The Sturm comparison theorem and its generalizations play an important role in the study of the oscillatory character of the second order linear equation

$$
y^{\prime \prime}+q(t) y=0, q \in C[a,+\infty) .
$$

One of the simpler forms of the theorem states that if equation (1) is disconjugate on $[a,+\infty)$ (that is, no solution of (1) has more than one zero on $[a,+\infty)]$, and if $q_{1} \in C[a,+\infty)$ with $q_{1}(t) \leq q(t)$ on $[a,+\infty)$, then the equation

$$
y^{\prime \prime}+q_{1}(t) y=0
$$

is also disconjugate on $[a,+\infty)$. This result may be extended to comparisons of an integral type, one of which is the so-called HilleWintner comparison theorem.

THEOREM 1 [8], [15]. Let $Q(t) \equiv \int_{t}^{\infty} q(s) d s$ and $Q_{1}(t) \equiv \int_{t}^{\infty} q_{1}(s) d s$

Received 24 August 1979. Research supported in part by a grant from the National Research Council of Canada. 
exist with $0 \leq Q_{1}(t) \leq Q(t)$ on $[a$, $+\infty)$ and assume equation (1) is disconjugate on $[a,+\infty)$. Then so also is equation (2).

In this paper we shall be interested in extending an analogue of Theorem $I$ to the third order linear equations

$$
L y \equiv y^{\prime \prime \prime}+q(t) y=0
$$

and

$$
L_{1} y \equiv y^{\prime \prime \prime}+q_{1}(t) y=0 .
$$

We recall. that equation (3) is said to be disconjugate on an interval $I \subset[a,+\infty)$ in case no nontrivial solution has more than two zeros on $I$. Disconjugacy and its connection with oscillation and nonoscillation have been studied by many authors ( $c f$. Barrett [1], Hanan [7], Lazer [11], Etgen and Shih [3], [4], [5], Jones [9], [10], and the references therein). In particular, it has been shown in [13] (see also [7, Theorem 3.11]) that if $\hat{q}(t) \leq q_{1}(t) \leq q(t)$ and if $L y=y^{\prime \prime \prime}+q(t) y=0$ and $\hat{L} y=y^{\prime \prime \prime}+\hat{q}(t) y=0$ are both disconjugate on $[a,+\infty)$, then so is $L_{1} y=y^{\prime \prime \prime}+q_{1}(t) y=0$. This may be thought of as one analogue of the Sturm Comparison Theorem in the study of the oscillatory character of $L y=0$. In order to compare our results with other known criteria for disconjugacy, we recall that if $q(t) \geq 0$ on $[a,+\infty)$ and if $L y=0$ is disconjugate on $[a,+\infty)$, then $([7])$,

$$
\int^{\infty} t q(t) d t<+\infty .
$$

Further, if

$$
\int^{\infty} t^{2} q(t) d t<+\infty
$$

then $L y=0$ is disconjugate on $\left[t_{0},+\infty\right)$, some $t_{0} \geq a,([6])$. On the other hand, if for some $\delta, 0<\delta<1$, we have

$$
\int^{\infty} t^{1+\delta} q(t) d t=+\infty
$$

then $L y=0$ is oscillatory (that is, $L y=0$ has a solution which changes sign on each half-line $\left.\left[t_{0},+\infty\right)\right)([11])$. Finally, comparison 
with the Euler equation shows that $L y=0$ is (eventually) disconjugate if

$$
\limsup _{t \rightarrow \infty} t^{3} q(t)<2 / 3 \sqrt{3}
$$

and is oscillatory if

$$
\underset{t \rightarrow \infty}{\lim \inf } t^{3} q(t)>2 / 3 \sqrt{3}
$$

Additional criteria may be found in the references cited above and in the book of Swanson [14]. We remark also that $L y=0$ is disconjugate on an interval $I$ (finite or infinite) in case there exist $\alpha, \beta \in C^{2}(I)$ with $\alpha<\beta$ on $I$ and $\alpha^{\prime \prime}+f\left(t, \alpha, \alpha^{\prime}\right) \geq 0 \geq \beta^{\prime \prime}+f\left(t, \beta, \beta^{\prime}\right), \quad t \in I$, where $f\left(t, r, r^{\prime}\right)=3 r r^{\prime}+r^{3}+p(t)$, (that is, $\alpha, \beta$ are lower and upper solutions of the Riccati equation for $L y=0$ ) (see [2]).

\section{Statement and proof of the results}

THEOREM 2. Assume $L y=0$ is disconjugate on $[a,+\infty)$, and let $q, q_{1} \in C[a,+\infty)$ satisfy

$$
q(t) \geq 0, \quad q_{1}(t) \geq 0, \quad t \geq a .
$$

Assume further that

$$
Q(t) \equiv \int_{t}^{\infty} q(s) d s \text { and } Q_{1}(t) \equiv \int_{t}^{\infty} q_{1}(s) d s
$$

exist and satisfy

$$
Q_{I}(t) \leq Q(t), \quad t \geq a \text {. }
$$

Then $L_{1} y=y^{\prime \prime \prime}+q_{1}(t) y=0$ is disconjugate on $\left[t_{0},+\infty\right)$ for some $t_{0} \geq a$

If we relax the requirement that $q_{1}$ be nonnegative, we may establish

COROLLARY 3. Asszome $L y=0$ is disconjugate on $[a,+\infty), q \geq 0$, and assume that

$$
Q_{1}^{+}(t) \leq Q(t), \quad t \geq a,
$$

and 


$$
Q_{1}^{-}(t) \leq Q(t), \quad t \geq a
$$

where

$$
Q_{1}^{+}(t) \equiv \int_{t}^{\infty} q_{1}^{+}(s) d s \text { and } Q_{1}^{-}(t) \equiv \int_{t}^{\infty} q_{1}^{-}(s) d s
$$

and where $q_{1}^{+}(t) \equiv \max \left[0, q_{1}(t)\right], q_{1}^{-}(t) \equiv \max \left[0,-q_{1}(t)\right]$. Then $L_{1} y=0$ is disconjugate on $\left[t_{0},+\infty\right)$ for some $t_{0} \geq a$.

It is not difficult to see that Theorem 2 and Corollary 3 are sharp by considering the Euler equation

$$
y^{\prime \prime \prime}+\alpha t^{-3} y=0, \alpha \text { real constant, }
$$

which is disconjugate on $[a,+\infty)(a>0)$ if and only if $|\alpha| \leq 2 / 3 \sqrt{3}$. We shall give below an example whose disconjugate behaviour may not be inferred by any criteria known to the author.

For the case of a finite interval $I=[a, b]$, we have the following analogue of Theorem 2. This result is also related to the so-called Levin comparison theorems for the second-order equation (1) and (2) (see [12] and [14]). We recall that the adjoint equation of (3) is

$$
L^{*} y=y^{\prime \prime \prime}-q(t) y=0 \text {. }
$$

THEOREM 4. Assume $q \geq 0$ on $[a, b]$ and that the following conditions hold:

$$
\begin{cases}\int_{t}^{b} q_{1}^{+}(s) d s \leq \int_{t}^{b} q(s) d s, & a \leq t \leq b, \\ \int_{t}^{b} q_{I}^{-}(s) d s \leq \int_{t}^{b} q(s) d s . & \end{cases}
$$

Further, let $L^{*} y=0$ have a solution $y=y(t)$ satisfying

$$
y>0, y^{\prime}>0, y^{\prime \prime}<0, a \leq t \leq b .
$$

Then $L_{1} y=0$ is disconjugate on $[a, b]$.

Before proving the above results, we shall need to establish some properties of the nonoscillatory solutions of the adjoint equation (16) under the assumption $q \geq 0, q \neq 0$. Recall that $L^{*} y=0$ is 
disconjugate on an interval $I$ if and only if $L y=0$ is disconjugate on $I$ (cf. [7]). If $I=[a,+\infty)$ and $y=y(t) \neq 0$ is a nonoscillatory solution of $L^{*} y=0$, we may suppose that $y(t)>0$ for $t \geq t_{0}$. Since $y^{\prime \prime \prime}=q(t) y \geq 0, \quad t \geq t_{0}$, it follows that $y^{\prime \prime}$ is increasing on $\left[t_{0},+\infty\right)$ and hence $y^{\prime}$ can change from negative to positive values at most once in $\left[t_{0},+\infty\right)$. Therefore, either $y^{\prime}(t) \leq 0$ for all $t \geq t_{0}$ or there exists $t_{1} \geq t_{0}$ with $y^{\prime}(t)>0$ on $\left[t_{1},+\infty\right)$. Suppose then that $y^{\prime}(t) \leq 0$ for all $t \geq t_{0}$. Now since $y^{\prime \prime}(t)$ is increasing, we must also have either $y^{\prime \prime}(t) \leq 0$ or $y^{\prime \prime}(t)>0$ eventually. But if $y^{\prime \prime}(t) \leq 0$, then $y^{\prime}(t)$ is decreasing and hence $y^{\prime}(t) \leq \delta<0$ for all large $t$ and some $\delta<0$, which contradicts the fact that $y(t)>0$ on $\left[t_{0},+\infty\right)$. On the other hand, if $y^{\prime \prime}(t)>0$ eventually, then it follows that $y^{\prime \prime}(t) \geq n>0$ for all large $t$ and some $n>0$, which implies that $y^{\prime}(t)++\infty$, a contradiction. We may conclude, therefore, that we must have $y^{\prime}(t)>0$ on $\left[t_{1},+\infty\right)$ for some $t_{1} \geq t_{0}$. We sumarize the above remarks in

LEMMA 5. If $q(t) \geq 0$ and if $y=y(t) \neq 0$ is a nonoscizlatory solution of $L^{*} y=y^{\prime \prime \prime}-q(t) y=0$, then there exists $t_{1} \geq a$ such that

$$
y(t) y^{\prime}(t)>0 \text { on }\left[t_{1},+\infty\right) \text {. }
$$

The following result gives a sufficient condition for disconjugacy of $L^{*} y=0$ on an arbitrary interval $I=[a, b]$ or $[a,+\infty)$. In the infinite interval case, it is actually a special case of a result of Lazer [11, Theorem 2.1]. However, the proof given below is different.

LEMMA 6. Let $q \geq 0, q \neq 0$, on $I$ and assume there exists $a$ solution of $L^{*} y=0$ satisfying

$$
y(t)>0, y^{\prime}(t)>0, y^{\prime \prime}(t)<0, t \in I .
$$

Then $L^{*} y=0$ is disconjugate on $I$.

Proof. Given the solution $y$ of $L^{*} y=0$ satisfying (20), let $z$ be the solution of $L^{*} y=0$ with

$$
z(a)=0, z^{\prime}(a)=1, z^{\prime \prime}(a)=1,
$$

where $I=[a, b]$ or $[a, \infty)$. Then since $z^{\prime \prime \prime}=q z \geq 0$ on $I$, it 
follows that $z>0, z^{\prime}>0, z^{\prime \prime}>0, t>a$. Therefore, the function $W(t)=y z^{\prime}-z y^{\prime}$ is a solution of $L y=0$ satisfying $W(a)=y(a) z^{\prime}(a)>0$ and $W^{\prime}=y z^{\prime \prime}-z y^{\prime \prime}>0$ on $I$ so that $W>0$, $W^{\prime}>0$ on $I$. Therefore, $\alpha(t) \equiv 0<\beta(t) \equiv W^{\prime} / W$ are lower and upper solutions of the Riccati equation for $L y=0$ and hence $L y=0$ is disconjugate on $I$ ([2]), that is, $L^{*} y=0$ is disconjugate on $I$.

The converse of Lemma 6 is true, under an additional assumption, for the infinite interval case. This is a special case of a result of Lazer [11, Theorem 2.2] to which we refer for the proof:

LEMMA 7. Let $q(t) \geq 0$ and $q(t) \neq 0, I=[a,+\infty)$, and assume

$$
\int_{a}^{\infty} t^{4} q(t) d t=+\infty \text {. }
$$

Then $L^{*} y=0$ is disconjugate on $\left[t_{0},+\infty\right)$ for some $t_{0} \geq a$ if and only if there exists a nonoscizlatory solution $y=y(t)$ of $L^{*} y=0$ satisfying

$$
y(t)>0, y^{\prime}(t)>0, y^{\prime \prime}(t)<0 \text {.on }\left[t_{1},+\infty\right),
$$

for some $t_{1} \geq a$.

Whether condition (22) is necessary for the existence of a solution of $L^{*} y$ satisfying (23) under the assumption that $L^{*} y=0$ is disconjugate appears to still be an open question ([11]).

The proof of Theorem 2 will be given by considering an appropriate two dimensional nonlinear Riccati system. To that end, we make the following change of variable in the equation $L^{*} y=y^{\prime \prime \prime}-q(t) y=0$ :

$$
u_{1}=y^{\prime} / y, u_{2}=y^{\prime \prime} / y \text {, }
$$

to obtain the system

$$
\left\{\begin{array}{l}
u_{1}^{\prime}=u_{2}-u_{1}^{2}, \\
u_{2}^{\prime}=-u_{1} u_{2}+q .
\end{array} .\right.
$$

Thus, if $y$ is a nonoscillatory solution of $L^{*} y=0$ with, say $y(t)>0, y^{\prime}(t)>0$ for $t \geq t_{0}$, then $u_{1}, u_{2}$ defined by (24) satisfy (25) which becomes, after an integration from $t$ to $T$, 
Comparison theorems of Hille-Wintner type

181

$t_{0} \leq t<T<+\infty$,

$$
\left\{\begin{array}{l}
u_{1}(t)=u_{1}(T)+\int_{t}^{T}\left(u_{1}^{2}-u_{2}\right) d s, \\
u_{2}(t)=u_{2}(T)+\int_{t}^{T}\left(u_{1} u_{2}\right) d s-\int_{t}^{T} q d s .
\end{array}\right.
$$

We may now give the proof of Theorem 2 .

Proof of Theorem 2. The proof of the theorem will be separated into two cases, according to whether condition (22) does or does not hold. We shall also assume that $q_{1} \neq 0$ for all large $t$ (otherwise there is nothing to prove).

Case (i): $\int^{\infty} t^{4} q(t) d t=+\infty$.

Suppose that $q, q_{1}$, are as in the hypotheses of Theorem 2. Since disconjugacy of $L y=0$ is equivalent to disconjugacy of $L^{*} y=0$, let $y=y(t) \neq 0$ be a nonoscillatory solution of $L^{*} y=0$ satisfying (23) on $\left[t_{0},+\infty\right), t_{0} \geq a$. Defining $u_{1}, u_{2}$ as in (24), we see that $u_{1}>0$, $u_{2}<0$ on $\left[t_{0}, \infty\right)$ and since $y^{\prime}(t)$ is decreasing and $y(t)$ is increasing on $\left[t_{0}, \infty\right)$, it follows that $\lim _{t \rightarrow \infty} u_{1}(t)=0$. Likewise, $\lim _{t \rightarrow \infty} u_{2}(t)=0$. Hence, letting $T \rightarrow \infty$ in system $(26)$, we see that $u_{1}, u_{2}$ satisfy

(27)

$$
\left\{\begin{array}{l}
u_{1}(t)=\int_{t}^{\infty} g_{1}\left(u_{1}, u_{2}\right) d s, t \geq t_{0}, \\
u_{2}(t)=\int_{t}^{\infty} g_{2}\left(u_{1}, u_{2}\right) d s-Q(t), \quad t \geq t_{0},
\end{array}\right.
$$

where $Q(t)=\int_{t}^{\infty} q(s) d s$, and

(28)

$$
\left\{\begin{array}{l}
g_{1}\left(u_{1}, u_{2}\right)=u_{1}^{2}-u_{2} \\
g_{2}\left(u_{1}, u_{2}\right)=u_{1} u_{2} .
\end{array},\right.
$$

https://doi.org/10.1017/S0004972700006006 Published online by Cambridge University Press 
Notice that if $u_{1}>0, u_{2}<0$, then $g_{1}$ is increasing in $u_{1}$ and decreasing in $u_{2}$ and $g_{2}$ is decreasing in $u_{1}$ and increasing in $u_{2}$. Consider now the system corresponding to $L_{1} y=y^{\prime \prime \prime}+q_{1} y=0$ and its adjoint $L_{1}^{*} y=y^{\prime \prime \prime}-q_{1} y=0$ :

(29)

$$
\begin{cases}v_{1}(t)=\int_{t}^{\infty} g_{1}\left(v_{1}, v_{2}\right) d s, & \\ v_{2}(t)=\int_{t}^{\infty} g_{2}\left(v_{1}, v_{2}\right) d s-Q_{1}(t), & t \geq t_{0} .\end{cases}
$$

We show first that (29) has a solution defined on $\left[t_{0},+\infty\right)$ which is obtainable by successive approximations. To see this, define the sequences $\left\{v_{1 n}(t)\right\},\left\{v_{2 n}(t)\right\}$ by

(30)

$$
\left\{\begin{array}{l}
v_{10}(t) \equiv u_{1}(t), \quad t \geq t_{0}, \\
v_{1 n}(t)=\int_{t}^{\infty} g_{1}\left(v_{1 n-1}, v_{2 n-1}\right) d s, n \geq 1, \quad t \geq t_{0},
\end{array}\right.
$$

and

$$
\left\{\begin{array}{l}
v_{20}(t) \equiv u_{2}(t), \quad t \geq t_{0}, \\
v_{2 n}(t)=\int_{t}^{\infty} g_{2}\left(v_{1 n-1}, v_{2 n-1}\right) d s-Q_{1}(t), n \geq 1, \quad t \geq t_{0} .
\end{array}\right.
$$

By induction, using the monotoneity of $g_{1}$ and $g_{2}$, it follows that $\left\{v_{1 n}\right\},\left\{v_{2 n}\right\}$ are well-defined for all $t \geq t_{0}$ and satisfy

$$
v_{1 n+1}(t) \leq v_{1 n}(t) \leq u_{1}(t), v_{2 n+1}(t) \geq v_{2 n}(t) \geq u_{2}(t), \quad t \geq t_{0} .
$$

Furthermore, since $g_{1}\left(u_{1}(t), u_{2}(t)\right)>0>g_{2}\left(u_{1}(t), u_{2}(t)\right)$, it follows (using $Q_{1}(t) \geq 0$ ) that

$$
v_{1 n}(t)>0>v_{2 n}(t), t \geq t_{0}, n=1,2, \ldots .
$$

Define $\hat{v}_{1}(t), \hat{v}_{2}(t)$ by 


$$
\begin{aligned}
& \text { Comparison theorems of Hille-Wintner type } \\
& \hat{v}_{1}(t) \equiv \lim _{n \rightarrow \infty} v_{1 n}(t), \hat{v}_{2}(t) \equiv \lim _{n \rightarrow \infty} v_{2 n}(t), t \geq t_{0} .
\end{aligned}
$$

It follows by the Monotone Convergence Theorem and Dini's Theorem that $\hat{v}_{1}(t), \hat{v}_{2}(t)$ solve system (29) on $\left[t_{0},+\infty\right)$, and that $\left\{v_{1 n}\right\},\left\{v_{2 n}\right\}$ converge uniformly on compact intervals to $\hat{v}_{1}, \hat{v}_{2}$. Further, since $q_{1}(t) \neq 0$, it follows that $\hat{v}_{2}(t)<0, t>t_{0}$. We may now define

$$
z(t)=\exp \int_{t_{0}}^{t} \hat{v}_{1}(s) d s, t \geq t_{0},
$$

and it follows that $z(t)$ is a solution of $y^{\prime \prime \prime}-q_{1} y=0$ on $\left[t_{0},+\infty\right)$ and satisfies $z(t)>0, z^{\prime}(t)=\hat{v}_{1}(t) z(t)>0$, and

$$
z^{\prime \prime}(t)=\left(\hat{v}_{1}^{2}+\hat{v}_{1}^{\prime}\right) z=\hat{v}_{2}(t) z(t)<0, \quad t \geq t_{0}
$$

Therefore, by Lemma 6, $L_{1}^{*} y=y^{\prime \prime \prime}-q_{1} y=0$ is disconjugate on $\left[t_{0},+\infty\right)$ and hence so also is $L_{1} y=y^{\prime \prime \prime}+q_{1} y=0$. This completes the proof for case (i).

Case (ii): $\int^{\infty} t^{4} q(t) d t<+\infty$.

In this case, we show that under the assumption that (12) holds (that is, $Q_{1}(t) \leq Q(t)$ ), it follows that

$$
\int^{\infty} t^{4} q_{1}(t) d t<+\infty
$$

so that condition (6) holds for $L_{1} y=0$ and therefore $L_{1} y=0$ is disconjugate ([5]). (The author is indebted to Professor G. Butler for the following observations.) Define $\mu(t)=t^{4}, t \geq a$. Then an integration by parts yields:

$$
\begin{aligned}
\int_{a}^{t} s^{4} q(s) d s & =\int_{a}^{t} \mu(s) q(s) d s \\
& =\int_{a}^{t} \mu^{\prime}(s) Q(s) d s-\mu(t) Q(t)+\mu(a) Q(a) .
\end{aligned}
$$


Similarly,

$$
\int_{a}^{t} s^{4} q_{1}(s) d s=\int_{a}^{t} \mu^{\prime}(s) Q_{1}(s) d s-\mu(t) Q_{1}(t)+\mu(a) Q_{1}(a) .
$$

Suppose, if possible, that $\int_{a}^{\infty} t^{4} q_{1}(t) d t=+\infty$. Then from (35) it follows that

$$
\lim _{t \rightarrow \infty} \int_{a}^{t} \mu^{\prime}(s) Q_{1}(s) d s=+\infty
$$

so that if we let

$$
\phi(t) \equiv \int_{a}^{t} \mu^{\prime}(s) Q(s) d s
$$

then $\lim _{t \rightarrow \infty} \phi(t)=+\infty$ (condition (12)). Now from (34), since

$\lim _{t \rightarrow \infty}[\phi(t)-\mu(t) Q(t)]$ is finite, there exists $c>0$ and $t_{0} \geq a$ so that

$$
\mu(t) Q(t) \geq \phi(t)+\mu(a) Q(a)-c, \quad t \geq t_{0},
$$

which implies

$$
\phi^{\prime}(t) \geq\left(\mu^{\prime}(t) / \mu(t)\right)\left(\phi(t)+c_{1}\right), \quad t \geq t_{0},
$$

where $c_{1}=\mu(\alpha) Q(a)-c$. Let $t_{1} \geq t_{0}$ such that $\phi(t) \geq 2\left|c_{1}\right|$ for $t \geq t_{1}$. Then integrating (38) from $t_{1}$ to $t \geq t_{1}$ we get

$$
\left(\phi(t)+c_{1}\right) /\left(\phi\left(t_{1}\right)+c_{1}\right) \geq \mu(t) / \mu\left(t_{1}\right), \quad t \geq t_{1},
$$

and so from (37) we have

$$
\mu(t) Q(t) \geq\left(\phi\left(t_{1}\right)+c_{1}\right)\left(\mu(t) / \mu\left(t_{1}\right)\right), t \geq t_{1},
$$

and hence

$$
Q(t) \geq\left(\phi\left(t_{1}\right)+c_{1}\right) / \mu\left(t_{1}\right)>0, t \geq t_{1},
$$

contradicting the integrability of $q$. Therefore, $\int_{a}^{\infty} t^{4} q_{1}(t) d t<+\infty$ and $L_{1} y=0$ is disconjugate. This completes the proof of Theorem 2 . 
Proof of Corollary 3. The proof of Corollary 3 follows immediately from Theorem 2. If the hypotheses of Corollary 3 hold, then it follows by Theorem 2 that both $y^{\prime \prime \prime}+q_{1}^{+} y=0$ and $y^{\prime \prime \prime}-q_{1}^{-} y=0$ are disconjugate on $\left[t_{0},+\infty\right)$. Since $-q_{1}^{-}(t) \leq q_{1}(t) \leq q_{1}^{+}(t)$, it follows ([7], Theorem 3.11, [13]) that $y^{\prime \prime \prime}+q_{1}(t) y=0$ is disconjugate on $\left[t_{0},+\infty\right)$.

Proof of Theorem 4. The proof of Theorem 4 is similar to the proof of Theorem 2 and Corollary 3. We consider system (26) with $t_{0}=a$ and $T=b$ and with $u_{1}, u_{2}$ defined as in (24), where $y$ satisfies (18). Assuming $q_{I} \geq 0$, then the corresponding system for $L_{1}^{*} y=0$ is

$$
\begin{cases}v_{1}(t)=\int_{t}^{b} g_{1}\left(v_{1}, v_{2}\right) d s+v_{1}(b), & \\ v_{2}(t)=\int_{t}^{b} g_{2}\left(v_{1}, v_{2}\right) d s-\hat{Q}_{1}(t)+v_{2}(b), & \end{cases}
$$

where $\hat{Q}_{1}(t)=\int_{t}^{b} q_{1}(s) d s$. We may now define the successive approximations $\left\{v_{1 n}\right\},\left\{v_{2 n}\right\}$ for $a \leq t \leq b$ by

$$
v_{10}(t)=u_{1}(t), v_{20}(t)=u_{2}(t),
$$

and

$$
\begin{aligned}
& v_{1 n}(t)=\int_{t}^{b} g_{1}\left(v_{1 n-1}, v_{2 n-1}\right) d s+u_{1}(b), \\
& v_{2 n}(t)=\int_{t}^{b} g_{2}\left(v_{1 n-1}, v_{2 n-1}\right) d s-\hat{Q}_{1}(t)+u_{2}(b) .
\end{aligned}
$$

The proof now proceeds as in Theorem 2 and we conclude that there exists a solution $z$ of $L_{1} y=0$ satisfying (20) so that $L_{1} y=0$ is disconjugate on $[a, b]$ by Lemma 6 . If now $q_{1} \geq 0$ is not assumed but condition (17) holds, then we argue as in Corollary 3 to show that $L_{1} y=0$ is disconjugate on $[a, b]$. This completes the proof of Theorem 4.

EXAMPLE 1. A special case of a result of Lazer [11, Theorem 3.5] 
implies that equation $(4)$ is disconjugate on $\left[t_{0},+\infty\right)$ for some $t_{0} \geq a$ in case $\int_{a}^{\infty} q_{1}(t) d t<+\infty, q_{1} \geq 0$ and $q_{1} \neq 0$ in any subinterval and provided the second order equation

$$
y^{\prime \prime}+\frac{3}{2}\left(\int_{t}^{\infty} q_{1}(t) d t\right) y=0
$$

is nonoscillatory. In particular, (44) is nonoscillatory (by comparison with $y^{\prime \prime}+\frac{1}{4} t^{-2} y=0$ ) in case

$$
\underset{t \rightarrow \infty}{\lim \sup } t^{2} \int_{t}^{\infty} q_{1}(t) d t<\frac{1}{4} \cdot \frac{2}{3}=\frac{1}{6} .
$$

Applying Theorem 2 with $q(t)=(2 / 3 \sqrt{3}) t^{-3}$ we conclude that $L_{1} y=0$ is disconjugate on $\left[t_{0},+\infty\right)$ for some $t_{0} \geq a$ in case $q_{1} \geq 0$ and

$$
\int_{t}^{\infty} q_{1}(t) d t \leq(1 / 3 \sqrt{3}) t^{-2}, \quad t \geq t_{0},
$$

which improves (45).

$$
\begin{aligned}
& \text { Thus, if } q_{1}(t)=k\left(1+\sin t^{\delta}\right) t^{-3}, \delta>0 \text {, then } \\
& \qquad \int_{t}^{\infty} q_{1}(s) d s=(k / 2) t^{-2}+o\left(t^{-2-\delta}\right), t \rightarrow \infty,
\end{aligned}
$$

so that if $\frac{1}{3}<k<2 / 3 \sqrt{3}$, then (46) holds for large $t$ and hence $L_{1} y=0$ is disconjugate on $\left[t_{0},+\infty\right)$ for some $t_{0} \geq a$ by Theorem 2 .

However, equation (44) is oscillatory since $\frac{3}{2} \int_{t}^{\infty} q_{1}(s) d s>\frac{1}{4} t^{-2}$ for large $t$. Thus, the criterion of Lazer is not applicable to this example nor do the conditions (6) or (8) hold.

EXAMPLE 2. If $q(t)=(2 / 3 \sqrt{3}) t^{-3}$, then $L^{*} y=y^{\prime \prime}-q y=0$ has a solution $y=y(t) \neq 0$ satisfying (2) on $I=[a, b]$ for all $0<a<b<+\infty$, (that is, $y(t)=t^{\lambda}$, where $0<\lambda<1$ ). Therefore, if $q_{1} \in C[a, b]$ and if 
(47)

$$
\begin{cases}\int_{t}^{b} q_{1}^{+}(s) d s \leq\left(t^{-2}-b^{-2}\right) / 3 \sqrt{3}, & \\ \int_{t}^{b} q_{1}^{-}(s) d s \leq\left(t^{-2}-b^{-2}\right) / 3 \sqrt{3}, & a \leq t \leq b,\end{cases}
$$

then it follows by Theorem 4 that $L_{1} y=0$ is disconjugate on $[a, b]$.

\section{References}

[1] John H. Barrett, "Oscillation theory of ordinary linear differential equations", Adv. in Math. 3 (1969), 415-509.

[2] Lynn Erbe, "Disconjugacy conditions for the third order linear differential equation", Canad. Math. Buzl. 12 (1969), 603-613.

[3] G.J. Etgen and C.D. Shih, "Disconjugacy and oscillation of third order differential equations with nonnegative coefficients", Proc. Amer. Math. Soc. 38 (1973), 577-582.

[4] G.J. Etgen and C.D. Shih, "On the oscillation of certain third order linear differential equations", Proc. Amer. Math. Soc. 41 (1973), 151-155.

[5] G.J. Etgen and C.D. Shih, "Conditions for the nonoscillation of third order differential equations with nonnegative coefficients", SIAM J. Math. Anal. 6 (1975), 1-8.

[6] Thomas G. Hallam, "Asymptotic behavior of the solutions of an $n$th order nonhomogeneous ordinary differential equation", Trans. Amer. Math. Soc. 122 (1966), 177-194.

[7] Maurice Hanan, "Oscillation criteria for third-order linear differential equations", Pacific J. Math. 11 (1961), 919-944.

[8] Einar Hille, "Non-oscillation theorems", Trans. Amer. Math. Soc. 64 (1948), 234-252.

[9] Gary D. Jones, "Properties of solutions of a class of third-order differential equations", J. Math. Anal. Appl. 48 (1974), 165-169. 
[10] Gary D. Jones, "An asymptotic property of solutions of $y^{\prime \prime \prime}+p y^{\prime}+q y=0$ ", Pacific J. Math. 47 (1973), 135-138.

[11] A.C. Lazer, "The behavior of solutions of the differential equation $y^{\prime \prime \prime}+p(x) y^{\prime}+q(x) y=0$ ", Pacific J. Math. 17 (1966), 435-466.

[12] A.Yu. Levin, "A comparison principle for second-order differential equations", Soviet Math. Dokl. 1 (1960), 1313-1316.

[13] A.Ju. Levin, "Some problems bearing on the oscillation of solutions of linear differential equations", Soviet Math. Dokl. 4 (1963), $121-124$.

[14] C.A. Swanson, Comparison and oscillation theory of linear differential equations (Mathematics in Science and Engineering, 48. Academic Press, New York and London, 1968).

[15] Aurel Wintner, "On the comparison theorem of Kneser-Hille", Math. Scand. 5 (1957), 255-260.

Department of Mathematics, University of Alberta,

Edmonton,

Alberta T6G 2GI,

Canada. 